\title{
A Functional Gamma-Band Defined by Stimulus-Dependent Synchronization in Area 18 of Awake Behaving Cats
}

\author{
Markus Siegel and Peter König \\ Institute of Neuroinformatics, Swiss Federal Institute of Technology and University of Zürich, CH-8057 Zürich, Switzerland
}

\begin{abstract}
During the past decade, numerous studies have demonstrated stimulus-specific synchronization of neuronal activity in the $\gamma$-frequency range. However, it appears that the different analyses are based on widely different assumptions about which frequency range to investigate. Therefore, the term " $\gamma$-synchronization" refers to an inhomogeneous spectrum of definitions and corresponding frequency bands. Moreover, most studies have been performed in anesthetized animals or in awake animals by use of fixation paradigms. Thus, it is difficult to relate these results to alert animals behaving under natural conditions. Here, we investigate stimulus specific synchronization in primary visual cortex of awake cats in a tracking paradigm. We record local field potentials and multiunit activity simultaneously from multiple electrodes. (1) We demonstrate that visual stimulation induces neuronal synchronization in a broad frequency range reaching well above $100 \mathrm{~Hz}$. (2) We derive a functional $\gamma$-band based on an objective criterion: We show that synchronization of neuronal activity is optimally orientation-tuned when a broad frequency band is considered. This band starts above $40 \mathrm{~Hz}$, a frequency that is typically related to the term $\gamma$-synchronization, and extends to very high frequencies. Interestingly, the frequency of maximum synchronization is different from the frequency at which synchronization is most stimulus specific. (3) We demonstrate synchronization of neuronal activity in a distinct low-frequency band with different properties suggesting separate functional roles of low- and highfrequency synchronization.
\end{abstract}

Key words: $\gamma$ oscillation; synchronization; primary visual cortex; awake behaving cat; area 18; local field potential; multiunit activity; tracking task

\section{Introduction}

Much attention has been paid to the temporal structure of neuronal activity. A notable contribution was a result of the idea that neuronal synchronization serves as a temporal code in cortical information processing (Abeles, 1982; Singer, 1999). Numerous studies have demonstrated high-frequency oscillatory synchronizations of neuronal activity in various species, cortical systems, and experimental paradigms (Eckhorn, 1994; König and Engel, 1995; Singer and Gray, 1995; Steriade, 1999; Hatsopoulos et al., 2001; Varela et al., 2001). Because most studies fall back on the frequency classification traditionally used in EEG analysis, the described high-frequency synchronizations are often called $\gamma$-synchronizations. However, several problems are associated with this approach. First, comparing different studies, it becomes obvious that analysis is not based on a common definition. Some authors refer to narrow frequency bands (e.g., 37-43 Hz) (Miltner et al., 1999), 35- $45 \mathrm{~Hz}$ (Llinas and Ribary, 1993; Rodriguez et al., 1999), 32-48 Hz (Fell et al., 2001), and 39-63 Hz (Fries et al., 1997), whereas others base their analysis on wider frequency bands, such as $30-70 \mathrm{~Hz}$ (Tallon-Baudry et al., 1997), 35-80 Hz (Kruse and Eckhorn, 1996; Brosch et al., 1997), 15-75 Hz (Destexhe et al., 1999), 40-120/50-150 Hz (Chrobak and Buzsaki, 1998), 20-100 Hz (von Stein et al., 2000), and 8-100 Hz (Ohl et al., 2001). As a consequence, the term $\gamma$-synchronization essen-

\footnotetext{
Received Sept. 27, 2002; revised Feb. 10, 2003; accepted Feb. 20, 2003.

This work was supported by Swiss National Science Foundation Grant 31-65415.01. We thank Dr. Johannes Sarnthein for technical assistance and helpful discussions.

Correspondence should be addressed to Markus Siegel, Institute of Neuroinformatics, University/ETH Zürich Winterthurerstrasse 190, CH-8057 Zürich, Switzerland. E-mail: msiegel@ini.phys.ethz.ch.

Copyright $\odot 2003$ Society for Neuroscience $\quad$ 0270-6474/03/234251-10\$15.00/0
}

tially refers to a rather inhomogeneous collection of definitions and corresponding frequency bands. Because analysis often averages over such arbitrarily chosen frequency bands or focusing on peak responses in those bands, it remains difficult to compare the results of different studies. Second, many of these studies have been performed using anesthetics that potentially influence the frequency spectrum of electrical signals generated by the cortex. Third, those studies investigating the temporal dynamics in awake animals rely primarily on fixation paradigms. However, under such conditions, the gaze is not perfectly stable, but microsaccades frequently occur. It has recently been shown that this type of eye movement influences the temporal structure of neuronal activity in primary visual cortex strongly (Martinez-Conde et al., 2000). These issues leave open the question of to what extent various results on neuronal activity in the $\gamma$-frequency range can be transferred to awake animals behaving under more natural viewing conditions.

These problems motivate the two central questions addressed by the present study: What is the frequency distribution of $\gamma$-synchronizations in awake behaving animals? Which frequency band can be derived as a $\gamma$-band on the basis of objective and functional criteria? To address these questions, we record local field potentials (LFPs) and multiunit activities (MUAs) in primary visual cortex (area 18) of the alert cat. Recordings are made simultaneously from up to 17 chronically implanted electrodes, whereas visual stimulation is controlled in a tracking paradigm. We study synchronization of neuronal activity on a short and long spatial scale by means of the spectral power of the LFP and the coherence between LFPs recorded simultaneously at different cortical sites. Eventually, the analysis of spike-field coher- 
ence allows us to relate the frequency distribution of synchronizations revealed by the LFP directly to neuronal spiking activity.

\section{Materials and Methods}

Behavioral procedure. Animals $(n=4)$ were placed in a box $(30 \times 30 \times 80$ $\mathrm{cm}^{3}$ ) constructed as a Faraday cage. One side of the box was transparent and facing a monitor (Sony MultiScan 17seII, $105 \mathrm{~Hz}$ vertical refresh rate) (Sony, Tokyo, Japan) on which the visual stimuli are displayed. As soon as the cats were paying attention to the display, a trial was started by showing a randomly moving target on a uniform gray background [a filled white circle; diameter, $1.1^{\circ}$; speed, $16.3 \pm 7.2^{\circ} / \mathrm{sec}(\mathrm{SD})$; direction of motion chosen randomly from an uniform distribution]. Animals were trained to track this target until it switched its shape to a square, which indicated the end of a trial. They were rewarded with liquefied cat food only if they tracked the target continuously for the full length of the trial. If a cat stopped tracking before the switch of the target shape, the animal was not rewarded, and the trial was aborted and excluded from additional analysis. The cats' behavior was monitored online using a small CCD camera (Conrad Electronics, Hirschau, Germany) mounted inside the box in front of the cat's head. A half-reflective mirror was placed between the camera and the cat's head, which allowed direct observation of the direction of gaze of the animal superimposed on the stimulus currently displayed on the monitor. After 2-4 weeks of training, the animals performed the tracking task reliably. Because of the superposition of the stimulus and the cat's head, the judgment of the cat's behavior was subjectively very obvious, making it easy to ensure a good tracking performance of the animal. Moreover, the high quality of tracking was indicated by the fact that using small patches of gratings (Gabor patches) as visual stimuli, the reverse correlation technique allowed receptive field estimation. Finally, we performed control recordings with the cats under anesthesia to confirm the characterization of the neuronal response properties and receptive field positions.

After training was completed, the visual stimuli that drive the recorded neurons were introduced into the experimental protocol. These stimuli consisted of sine-wave gratings of 12 different orientations (spatial frequency, 0.44 cycles per degree) displayed as a static background covering the entire monitor display (horizontal size, $52^{\circ}$; vertical size, $44^{\circ}$ ). Stimuli were flashed behind the target $\geq 1 \mathrm{sec}$ after trial onset and kept displayed until the end of the trial (either reward or trial abort). The position of the target was kept inside the central area of the stimulus screen (radius, $15^{\circ}$ ) to prevent placement of receptive fields outside the stimulus area.

Electrodes and data acquisition. Electrode arrays consisted of Tefloncoated platinum iridium wires with a diameter of $50 \mu \mathrm{m}$ and an impedance of $\sim 300 \mathrm{k} \Omega$ at $1 \mathrm{kHz}$. Multiple electrodes (two cats with 16 electrodes; two cats with 17 electrodes) were implanted chronically in one hemisphere of each animal in area 18 close to the vertical meridian in the lower visual field. Electrodes were placed in an orthogonal array oriented in a sagittal plane, with a grid spacing of $\sim 1 \times 0.6 \mathrm{~mm}$ and with a minimum interelectrode distance of $\sim 0.6 \mathrm{~mm}$ and a maximum interelectrode distance of $\sim 4 \mathrm{~mm}$. As a consequence, the placement of the electrodes in different layers was evenly distributed in supragranular, granular, and deep cortical layers. In addition, two silver balls were placed epidurally for ground and reference. The raw data from all implanted electrodes were recorded simultaneously at $20 \mathrm{kHz}$ sampling rate together with stimulus triggers (SynAmps amplifier; Neuroscan Laboratories, Sterling, VA). The LFP and MUA were derived offline: The LFP is the low-frequency component of the recorded broadband signal and is obtained by low-pass filtering (cutoff $200 \mathrm{~Hz}$ ) and resampling the raw data at $400 \mathrm{~Hz}$. Accordingly, analysis in the frequency domain was performed up to $200 \mathrm{~Hz}$. The MUA is derived by high-pass filtering the raw data with a cutoff at $500 \mathrm{~Hz}$ and detecting unidirectional crossings of a manually placed threshold as spike events. The MUA spike trains were eventually stored with a temporal resolution of $1 \mathrm{msec}$. All experimental procedures were in accordance with the Kantonal Law for the protection of Experimental Animals and conformed with National Institutes of Health and Society for Neuroscience (United States) regulations.

Data analysis. For comparison of neuronal activity before stimulus onset and during visual stimulation, two time windows were analyzed: The first window (delay period) ranged from $500 \mathrm{msec}$ before to the time of stimulus onset. The second window (stimulus period) ranged from 200 to $700 \mathrm{msec}$ after stimulus onset. In the first $200 \mathrm{msec}$ after onset, transient responses were observed, which were not in the scope of the present study. However, these transient responses were well depicted in the sliding-window analyses, which were also performed for the LFP power and LFP coherence (100 msec Hanning window shifted with 1 msec step size) (see Figs. 1, 4). LFP power spectra were computed using standard algorithms (Press et al., 1992), known as Welch's method. The LFP coherence between two recorded signals $x$ and $y$ as a function of the given frequency $f$ is calculated as follows:

$$
\operatorname{Coh}_{x y}(f)=\left|P_{x y}(f)\right|^{2} /\left[P_{x x}(f) \times P_{y y}(f)\right],
$$

where $P_{x y}(f), P_{x x}(f)$, and $P_{y y}(f)$ are cross-spectra and auto-spectra of the signals $x$ and $y$, respectively. During the prestimulus period, a small peak was detected in the LFP power at $105 \mathrm{~Hz}$ related to the monitor refresh rate. It was on the order of $2 \%$ of the total signal power during the delay period. Although this seems negligible, the computed relative response strengths of LFP power and coherence in the respective frequency bins were strongly biased. Therefore, we discarded these bins and used the values of the neighboring entries. For the LFP power response and coherence response (see Figs. 1, 2, 4, 5), spectra were averaged over all stimulus orientations. To test the trial-to-trial variability of the LFP power response and coherence response, the response function averaged over all trials was fitted with a Gaussian function in the range from 25 to $100 \mathrm{~Hz}$ by $\chi^{2}$ minimization (see Figs. 2, 5). Accordingly, the response functions of the power and coherence for all individual trials were fitted with a Gaussian function (see Figs. 2, 5). To test whether the peak of the averaged response could be a sum of sharper response peaks in individual trials, the distribution of peak widths of individual trials was tested against the peak width of the response averaged over all trials (sign test; $p=0.01$ ). The phase coupling between the frequency components $f_{1}$ and $f_{2}$ of the signal $x$ was estimated by computing the bicoherence as follows:

$$
\operatorname{BiCoh}_{x}\left(f_{1} f_{2}\right)=\left|B_{x}\left(f_{1} f_{2}\right)\right|^{2} /\left[P_{x}\left(f_{1}\right) \times P_{x}\left(f_{2}\right) \times P_{x}\left(f_{1}+f_{2}\right)\right],
$$

where $B_{x}\left(f_{1}, f_{2}\right)$ and $P_{x}(f)$ are bispectra and power spectra of the signal $x$, respectively. The tuning index, which describes the goodness of tuning as a function of frequency, was computed for the LFP power and LFP coherence by vector averaging the response vectors for all stimulus orientations. The length of the resulting vector was then divided by the mean variance of the responses for all orientations as follows:

$$
\text { tuning index }=\left|\Sigma_{\phi} r(\phi) \times e^{i 2 \phi} / n_{\phi}\right| /\left[\Sigma_{\phi} \operatorname{var}[r(\phi)] / n_{\phi}\right],
$$

where $r(\phi)$ is the response to the stimulus of the orientation $\phi$. The resulting tuning index indicates which frequency of the LFP power has to be considered for optimal signal-to-noise ratio with respect to orientation tuning (see Figs. 3, 6). This analysis is performed for the responses in all possible frequency bands defined by $f_{\text {start }}$ and $f_{\text {stop }}$ by pooling data of all frequencies $f$ with $f_{\text {start }} \leq f<f_{\text {stop }}$. The significance of orientation tuning was tested by comparing the response to the optimal stimulus orientation against the response to the orthogonal orientation (Wilcoxon test; $p=0.01$ ). The tuning index calculated this way depends on two parameters: the difference between response to preferred and antipreferred orientation (response strength) and the width at half-height of the tuning curve (the response specificity) (Swindale, 1998). To investigate these parameters, we also separately computed indices for the response strength [(preferred response - antipreferred response)/variance of response] and tuning specificity [length of averaged response vector/(mean response - antipreferred response)]. However, these indices yielded very similar results. Thus, we depict only the results for the described tuning index, which sensibly combines these two underlying parameters. The global maximum of the tuning index computed for all possible frequency bands indicates which continuous frequency band has to be considered for optimal signal-to-noise ratio with respect to orientation tuning of the neuronal synchronization. However, the tuning index computed for single-frequency bins is also displayed to show the frequency distribution of tuning and reveal local maxima, which are not necessarily included in the continuous optimal band. Local maxima of the tuning index indicate separate bands of orientation tuning, which may be nonoverlapping with 
A

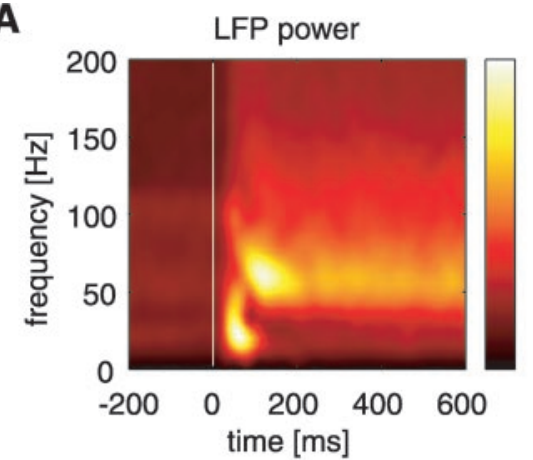

D

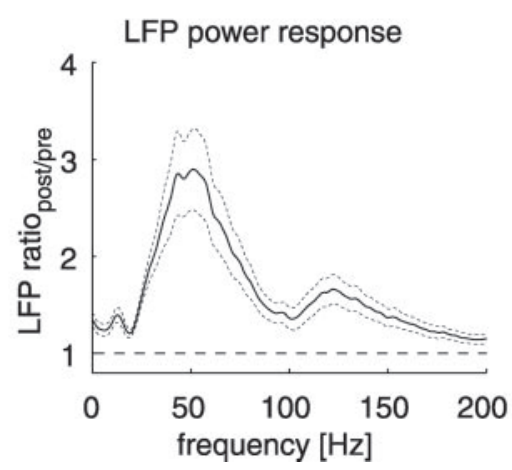

B
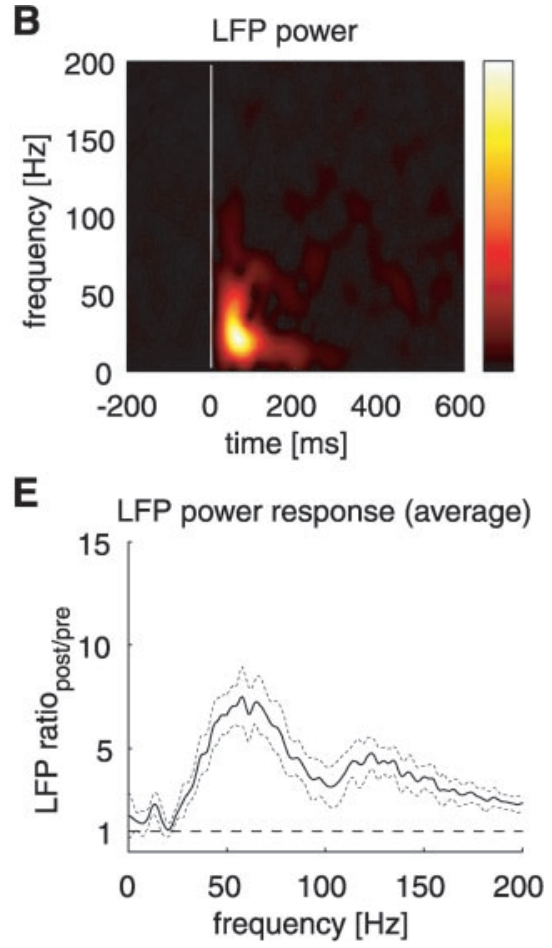

C
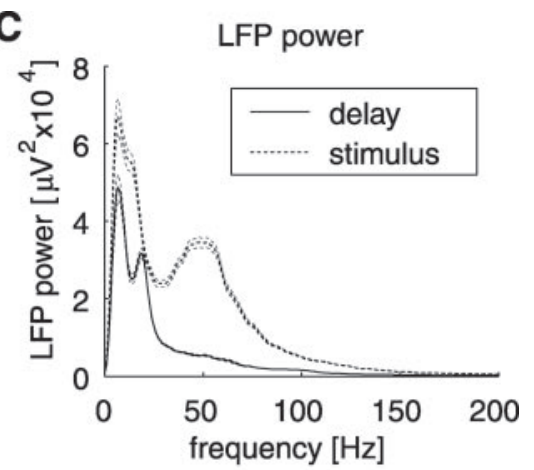

Figure 1. LFP power response. $A$, Time course of the spectral power of the LFP revealed by sliding-window analysis. The vertical white line indicates stimulus onset. To enhance readability, the power is normalized by 1 /frequency. $B$, The sliding-window power spectrum of the LFP after averaging the LFP over all trials, which matches the sliding-window power spectrum of the visual evoked potential. To enhance readability, the power is normalized by 1 /frequency (same recording site and data set). C, Power spectra of the LFP during the delay period ( -500 msec up to stimulus onset) and during the stimulus period ( $200-700$ msec past stimulus onset; same data set). D, The response in LFP power induced by visual stimulation, which equals dividing the dashed line by the solid line in C (same data set). The dashed line indicates the level at which no response would be measured. The dotted lines indicate mean \pm SEM. $E$, Grand average of the LFP power responses of all recording sites that showed a significant response $(n=55$; mean \pm SEM).

the optimal band indicated by the global maximum. The spike-triggered average (STA) was computed by cross-correlating MUA and LFP normalized by the spike rate of the MUA (see Fig. 7). The spike-field coherence (SFC) was then obtained by normalizing the power spectrum of the STA by the power spectrum of the LFP used to calculate the STA. The SFC as a function of frequency $f$ thus ranged from 0 to 1 and described the fraction of spikes phase-locked to the frequency component $f$ of the LFP. Thereby, the SFC was independent of the LFP power and the spike rate. The response functions for LFP power, LFP coherence, and SFC were calculated by dividing (LFP power) or subtracting (LFP coherence, SFC) the corresponding spectra for the delay and stimulus periods averaged over all stimulus orientations. The significance of the resulting response functions was tested by comparing the measures of the delay period and the stimulus period at the frequency of maximum response (Wilcoxon test; $p=0.01$ ).

\section{Results}

Short-range synchronization: frequency distribution and optimally tuned frequency band

To investigate the short-range synchronization of neuronal activity, we analyzed the spectral power of the LFP at 66 recording sites. Because the LFP reflects the summed activity of a local pool of neurons, large amplitudes of the LFP indicate synchronous activity within a small neighborhood of the recording electrode (Abeles, 1982; Engel et al., 1990). Thus, the spectral power of the LFP is a suitable measure reflecting correlated activity of a population of neurons in a region spanning a few hundreds of micrometers and sharing similar response properties.

At most recording sites, visual stimulation induced a characteristic response of the LFP power spectrum. The time course of this response was analyzed by the sliding-window technique (Fig. $1 A$ ) (see Fig. $7 B$ for a single-trial raw data trace of the LFP). Two distinct components could be easily identified. The early peak in low frequencies shows up in the sliding-window power spectrum of the visual evoked potential (VEP) (Fig. $1 B$ ) and thus reflects the stimulus-locked component of the response. In contrast, the second, slightly delayed response at high frequencies is not visible in the time course of the VEP power spectrum and thus is not phase-locked to stimulus onset. This high-frequency response exhibits a transient peak followed by a stable tonic response. To characterize the frequency distribution of this response, we computed the LFP power separately for the delay ( $-500 \mathrm{msec}$ up to stimulus onset) and stimulus period (200-700 msec past stimulus onset) (Fig. 1C, same data set). For the example shown, the relative change in power caused by visual stimulation was maximal at $58 \mathrm{~Hz}$ (Fig. 1D). However, the frequency distribution of this response was not centered sharply around the peak frequency but rather was broad and long-tailed toward high frequencies $>100 \mathrm{~Hz}$. At the channels in which significant responses were observed $(p<0.01 ; n=55$; total $=66 ; 83 \%)$, the frequency distributions of the LFP power responses were very similar. We averaged all significant examples to obtain the mean LFP power responses shown in Figure $1 E$. This mean response function shows the same characteristics as the example described above: a small response is visible in the low-frequency range up to $27 \mathrm{~Hz}$. It rises steeply, peaks at $43 \mathrm{~Hz}$, and shows a rather broad distribution up to high frequencies $>100 \mathrm{~Hz}$. For statistical comparison, we computed the frequencies of maximum response of all averaged curves. The median of these frequencies was $44 \mathrm{~Hz}$ (25th and 75th percentiles, 29 and $50 \mathrm{~Hz}$ ). The median of the response ratios at these frequencies was 2.1 (25th and 75th percentiles, 1.7 and 3.0), which corresponds to an increase of the LFP 
power by $\sim 100 \%$. The comparatively small low-frequency response is indicated by a local maximum at $13 \mathrm{~Hz}$ (Fig. 1E). A significant response at this frequency was found for $\sim 35 \%$ of all investigated recording sites $(p<0.01 ; n=23$; total $=66 ; 35 \%)$.

The slow decline of the power response toward higher frequencies was not monotonic but rather displayed a local maximum beyond $100 \mathrm{~Hz}$ (Fig. $1 \mathrm{E}$ ). To investigate the possibility of a harmonic effect, we analyzed the phase coupling between different frequencies by means of the bicoherence estimate. A significant phase coupling of the $120 \mathrm{~Hz}$ component to lower-frequency $\gamma$ rhythms was found for approximately one-third of the recording sites during the stimulus period $(p<0.01 ; n=21$; total $=66$; $32 \%)$. The median of the frequencies at which phase coupling was maximal is $56 \mathrm{~Hz}$. However, the absolute values of bicoherence are very low (median $=0.08 ; 25$ th and 75 th percentiles, 0.03 and $0.1 ; n=31)$. Although a harmonic effect cannot be ruled out, the low incidence of a significant effect and the low absolute values render a harmonic cause of the local maximum $>100 \mathrm{~Hz}$ very unlikely.

The broad frequency distribution of the LFP power response demonstrated in Figure 1 raises the question of whether it is because of the superposition of sharp peaks in the responses of individual trials shifted to different frequencies or whether individual trials show similar broad distributions. To answer this question, we analyzed the trial-to-trial variability of the LFP response for all recording sites that showed a significant response. Figure 2 displays the results for the same data set as that examined in Figure $1 A-D$. To parameterize the properties of the LFP power response, a Gaussian function was fitted to the central peak of the response function averaged over all trials (Fig. $2 \mathrm{~A}$, bottom trace). The same was done individually for all the single-trial response functions of the LFP power (Fig. 2A, top traces represent eight randomly chosen trials). To answer the question of whether the trial-to-trial variability accounts for the broad peak of the averaged response, we compared the distribution of the peak frequency and peak width of the single-trial fits with the corresponding parameters of the fit to the average response function. Although the peak frequencies of the fits for individual trials are distributed around the peak frequency of the fit to the average response function (Fig. $2 \mathrm{~B}$ ), the median of the peak width for individual trials is significantly smaller than the peak width of the fit to the average response function (Fig. $2 C)(p<0.01)$. The same is true for all investigated recording sites that showed a significant response $(p<0.01 ; n=55$; total $=55 ; 100 \%)$. Thus, the trial-to-trial variability of the position of relatively sharper response peaks accounts for the broad frequency distribution of short-range synchronization induced by visual stimulation.

Given the demonstrated broad frequency distribution of short-range synchronization in response to visual stimulation, can objective and functional criteria be used to define the frequency band that yields the optimal signal-to-noise ratio with respect to the displayed stimulus? Because we used gratings of different orientations for visual stimulation, we analyzed in which frequency band local synchronization of neuronal activity, reflected by the LFP power, was optimally tuned for stimulus orientation. We therefore computed a tuning index that describes the goodness of tuning as a function of frequency (see Materials and Methods). For the example shown in Figure $3 A$, the maximal orientation tuning of a single frequency bin is found at $75 \mathrm{~Hz}$. To derive a frequency band of optimal orientation tuning rather than a single-frequency bin, we computed the tuning index as a function of all possible frequency bands defined by $f_{\text {start }}$ and $f_{\text {stop }}$. Thus, the LFP power at frequencies $f$ with $f_{\text {start }} \leq f<f_{\text {stop }}$ was

\section{A LFP power response}
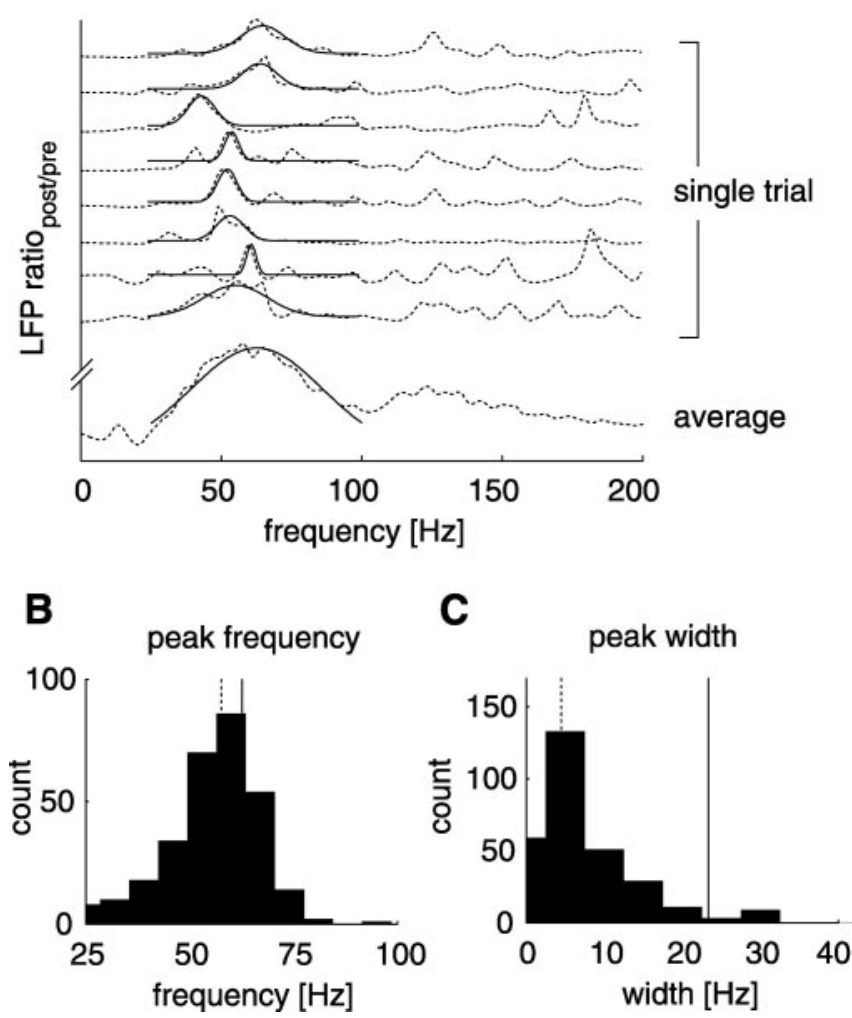

Figure 2. Single-trial analysis of the LFP power response. $A$, The bottom dashed trace shows the mean LFP power response averaged over all trials (same recording site and data set as in Fig. 1). The solid line indicates the best fit of a Gaussian function over the frequency range of 25-100 $\mathrm{Hz}$. The top eight dashed traces display the LFP responses of eight randomly chosen trials (same data set). The solid lines indicate the corresponding best Gaussian fits from $25-100 \mathrm{~Hz}$. B Distribution of the peak frequencies of the Gaussian fits of all trials. The dashed line indicates the median of the distribution ( $n=297$; median $=58 \mathrm{~Hz}$, same recording site). The solid line indicates the peak frequency of the Gaussian fit to the averaged LFP response displayed in $A$ ( 63 $\mathrm{Hz}$ ). C, Distribution of the peak width of the Gaussian fits of all trials (SD of the fitted Gaussian). The dashed line indicates the median of the distribution ( $n=297$; median $=4.5 \mathrm{~Hz}$, same recording site). The solid line indicates the peak width of the Gaussian fit to the averaged LFP response displayed in $A(23.3 \mathrm{~Hz})$.

averaged before computation of the tuning index (Fig. 3B). For the chosen example, the maximum tuning index (indicated by the diamond) was found for $f_{\text {start }}=64 \mathrm{~Hz}$ and $f_{\text {stop }}=103 \mathrm{~Hz}$. Interestingly, this band does not include the frequencies around $20 \mathrm{~Hz}$, at which a high tuning index is indicated by the local maximum in Figure $3 A$ and a corresponding local maximum in Figure $3 B$ for $f_{\text {start }}=15 \mathrm{~Hz}$ and $f_{\text {stop }}=32 \mathrm{~Hz}$. At this low frequency, the response is significantly tuned $(p<0.01)$, and the preferred orientation matches the optimal orientation of the optimal frequency band. However, it is not included in the optimal band because of the dip around $40 \mathrm{~Hz}$, which separates these lowand high-frequency bands. The same analysis of the tuning index was performed for each recording site, and the resulting optimal frequency band was tested for significant orientation tuning. Significant orientation tuning was found in 38 cases, which are investigated below $(p<0.01 ; n=38$; total $=66 ; 58 \%)$. The general characteristics are displayed in Figure 3, $C$ and $D$, which depict the tuning indices for the LFP power averaged over all significant recording sites. The tuning index of single-frequency bins increases steeply in the range of $45 \mathrm{~Hz}$ (Fig. 3C), which is consistent with a narrow distribution of $f_{\text {start }}$ found for all significant record- 


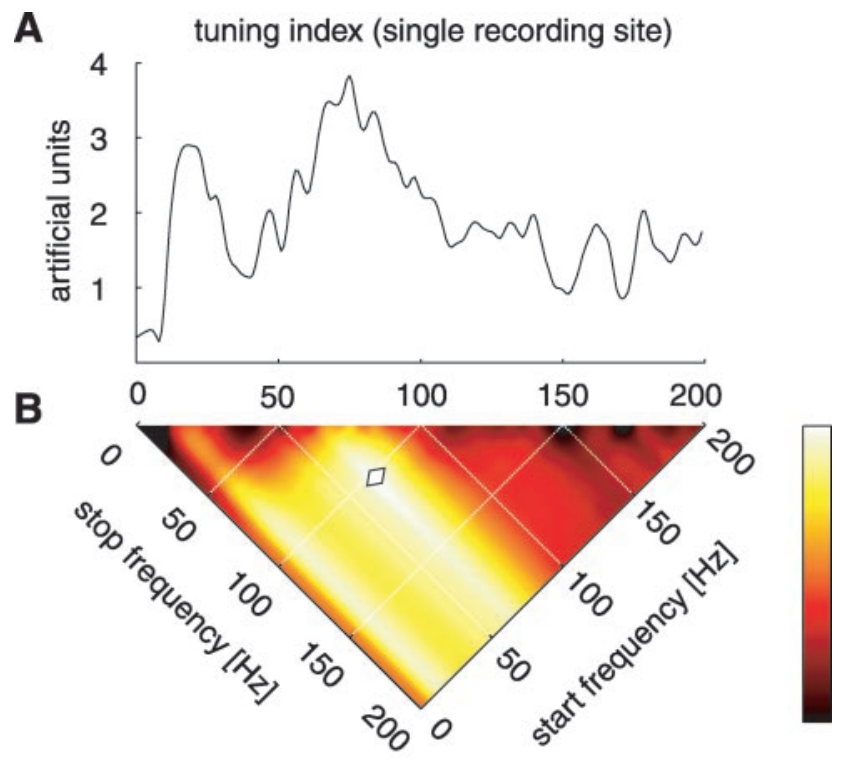

C tuning index (grand average)

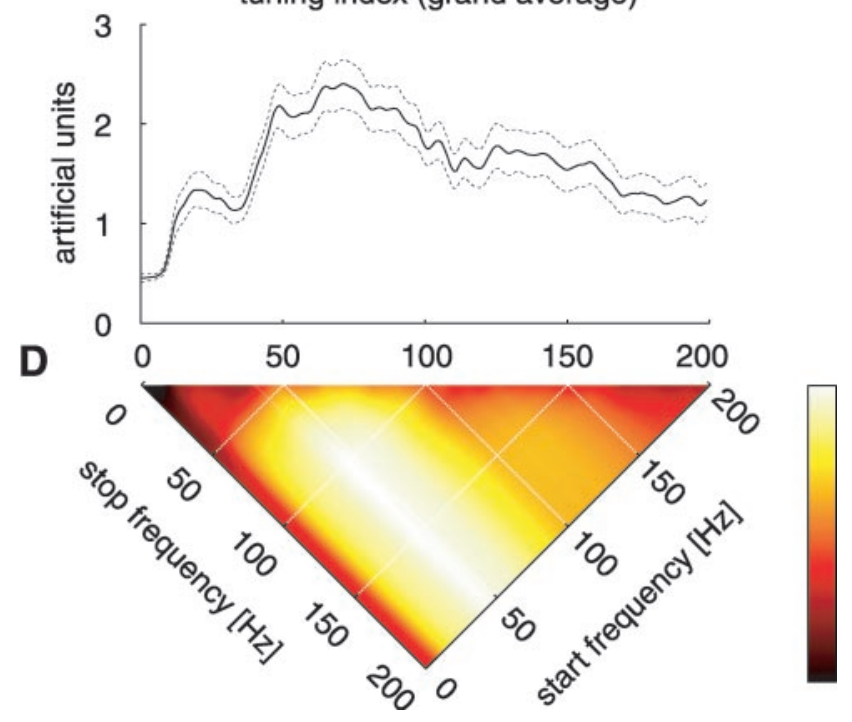

Figure 3. Orientation tuning index of the LFP power. $A$, The tuning index of a typical recording site. The tuning index displays the signal-to-noise ratio with respect to orientation tuning as a function of frequency. $B$, The same tuning index computed for all frequency bands defined by the start and stop frequency (same recording site and data set). The tuning index for the example shown is maximal taking into account the spectral power in the band ranging from $f_{\text {start }}=$ $64 \mathrm{~Hz}_{\text {to }} \mathrm{f}_{\text {stop }}=103 \mathrm{~Hz}$ (indicated by the diamond). The function displayed in $A$ is identical to the values on the diagonal of $B$. $C$, Grand average of the tuning index shown in $A$ over all recording sites that showed a significant orientation tuning $(n=38$; mean \pm SEM). $D$, Tuning index as a function of all frequency bands defined by $f_{\text {start }}$ and $f_{\text {stop }}(n=38)$. The function displayed in C is identical to the values in the diagonal of $D$.

ing sites (median $=45 \mathrm{~Hz} ; 25$ th and 75th percentiles, 39 and 62 $\mathrm{Hz} ; n=38$ ). It peaks at $\sim 70 \mathrm{~Hz}$ and shows a slow falloff toward higher frequencies, which can be understood from the broad distribution of $f_{\text {stop }}$ (median $=106.5 \mathrm{~Hz} ; 25$ th and 75th percentiles, 69 and $173 \mathrm{~Hz} ; n=38$ ) and the long-tailed distribution of the individual examples. These properties lead to the horizontal streak toward higher stop frequencies in Figure $3 D$, in which the tuning index does not vary greatly if higher frequencies are included. The distribution of the frequencies for maximum orientation tuning of single-frequency bins is centered around the median of $64 \mathrm{~Hz}$ (25th and 75th percentiles, 50 and $76 \mathrm{~Hz} ; n=$ 38). As demonstrated for the example shown in Figure $3, A$ and $B$, we observe a distinct peak in the low-frequency range from $\sim 10$ to $30 \mathrm{~Hz}$ displayed as a local maximum in Figure $3 C$ that corresponds to a local maximum in Figure $3 D$ for $f_{\text {start }}=15 \mathrm{~Hz}$ and $f_{\text {stop }}=33 \mathrm{~Hz}$. A distinct low-frequency band that shows significant orientation tuning and does not overlap with the optimal band was found in approximately one-third of the recording sites $\left(p<0.01 ; n=14\right.$; total $=38 ; 37 \% ;$ median $f_{\text {start }}=12 \mathrm{~Hz}$; median $f_{\text {stop }}=30 \mathrm{~Hz}$ ). Because this low-frequency band does not overlap with the optimal band found for the global maximum, it suggests a distinct low-frequency component of local synchronization of neuronal activity.

In summary, visual stimulation induces a prominent broadband increase of short-range synchronization. By analysis of optimal orientation tuning, the frequency band from 45 to $106.5 \mathrm{~Hz}$ is derived for short-range synchronization. Finding orientation tuning of short-range synchronization at such high frequencies is surprising, considering the comparatively low-frequency bands used by most other studies. Indeed, using a functional definition, such as optimal orientation tuning, results in a frequency band just above but not including the $40 \mathrm{~Hz}$ bin. This does not imply that activity at $40 \mathrm{~Hz}$ is not orientation-tuned in primary visual cortex of awake cats. It shows, however, that including higher frequencies leads to better orientation-tuned responses of the LFP rather than including the $40 \mathrm{~Hz}$ bin.

\section{Long-range synchronization: frequency distribution and optimally tuned frequency band}

To estimate long-range synchronization of neuronal activity, we computed coherence spectra of LFPs recorded simultaneously at different cortical sites. The coherence ranges from 0 to 1 , expresses the phase locking of two signals as a function of frequency, and is independent of changes in the total amount of power. Because the use of coherence spectra enables direct analysis in the frequency domain, we performed the analyses analogously to that demonstrated for the LFP power above.

In most investigated pairs of recording sites, visual stimulation induced an increase of LFP coherence similar to the response observed for the LFP power (Fig. 4A). The time course of the coherence revealed a transient peak in lower frequencies and a tonic increase of synchronization in higher frequencies. The shift-predictor coherence revealed synchronization between LFPs because of signal components that were phase-locked to stimulus onset (Fig. $4 B$ ). Here, only the transient low-frequency peak appeared, indicating that this component was caused by the VEP as observed for the LFP power. By subtracting coherence spectra during the delay period from that of the stimulus period (Fig. 4C), the coherence response is derived for the same example (Fig. 4D). The median of the maximum responses for all significant responses was 0.16 (25th and 75th percentiles, 0.09 and 0.24 ), which corresponds to an increase of the LFP coherence by $\sim 114 \%$. The median of the maximum-response frequencies of all significant pairs was $49 \mathrm{~Hz}$ (25th and 75th percentiles, 26.5 and $56 \mathrm{~Hz}$ ). The responses tested as significant $(p<0.01 ; n=244$; total $=512 ; 48 \%$ ) were averaged to obtain the mean frequency distribution of LFP coherence induced by visual stimulation (Fig. $4 E$ ). It peaks at $\sim 50 \mathrm{~Hz}$ and shows a long-tailed falloff toward higher frequencies similar to the response of the LFP power. However, in contrast to the response function of the LFP power, visual stimulation also induced a prominently increased coherence in the low-frequency range that showed up as a local maximum at $13 \mathrm{~Hz}$ in the average coherence response (Fig. 4E). A significant low-frequency response at this frequency was found for $\sim 22 \%$ of all investigated pairs of recording sites $(p<0.01$; 
A

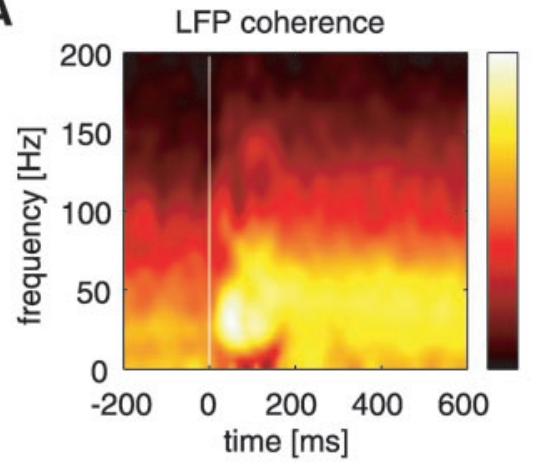

D

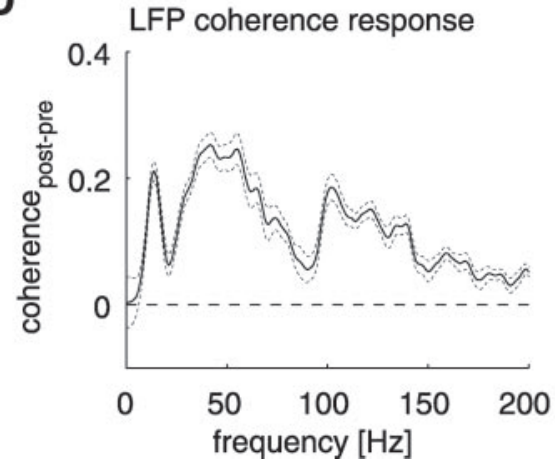

B

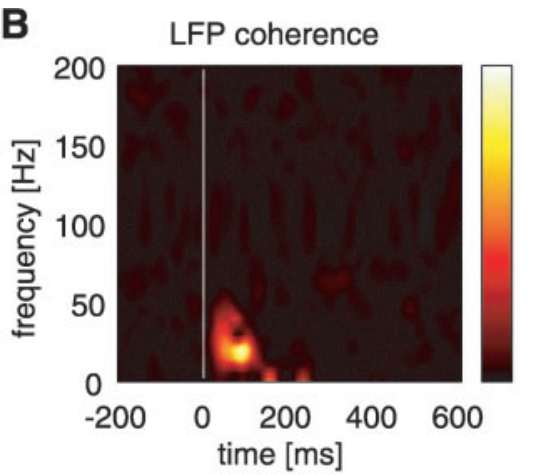

E

E LFP coherence response (average)

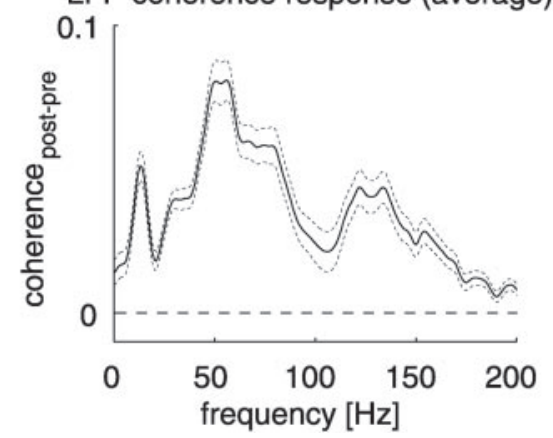

C
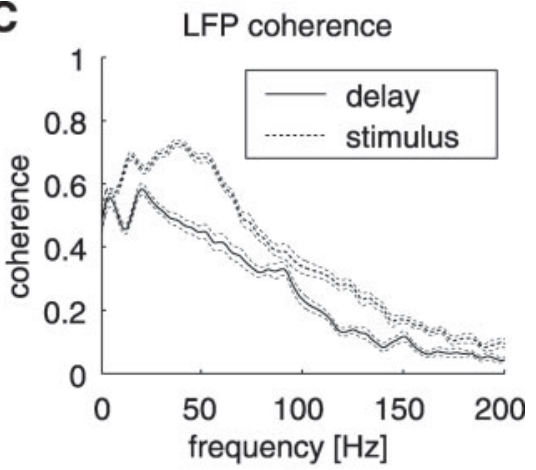

Figure 4. LFP coherence response. $A$, Time course of the coherence between two recording sites revealed by sliding window analysis. The vertical white line indicates stimulus onset. $B$, The sliding-window coherence of the shift predictor computed by independently shuffling trials of both recording sites. Thus, only coherence resulting from signal components that are phase locked to stimulus onset is revealed (same recording sites and data set). C, LFP coherence spectra during the delay period ( -500 msec up to stimulus onset) and during the stimulus period ( $200-700$ msec past stimulus onset; same data set). $D$, The response in LFP coherence induced by visual stimulation that is the difference between the two traces in $C$ (same data set). The dashed line indicates the level at which no response would be measured. The dotted lines indicate mean \pm SEM. $E$, Grand average of the LFP coherence responses of all pairs of recording sites that showed a significant coherence response $(n=244$, mean \pm SEM).

$n=112$; total $=512 ; 22 \%)$. A measure typically used in other studies is the frequency of strongest synchronization during stimulation without taking the delay period into account. Using this measure, for all pairs that showed a significant coherence response, the median of the frequencies of strongest synchronization was found at $43 \mathrm{~Hz}$, which agrees well with previous studies.

As demonstrated for short-range synchronization, we investigated whether the broad frequency distribution of the coherence response was a result of trial-to-trial variability in the position of sharper response peaks of individual trials (Fig. 5, same data set). The averaged coherence response (Fig. $5 A$, bottom trace) and the responses of individual trials (Fig. $5 A$, top eight traces represent eight randomly chosen trials) were fitted with a Gaussian function. The peak frequencies of the individual trial fits were well distributed around the peak frequency of the fit to the average response (Fig. $5 B$ ). However, the median of the peak widths of the individual trials was significantly smaller than the width of the average coherence response peak (Fig. $5 C)(p<0.01)$. The same is true for the vast majority of all investigated pairs of recording sites that showed a significant response $(p<0.01 ; n=211$; total $=244 ; 90 \%)$. Thus, the trial-to-trial variability of sharp response peaks accounts for the broad frequency distribution of LFP coherence induced by visual stimulation.

To investigate the influence of volume conduction, we compared the average coherence during the delay period for all pairs of recording sites as a function of the interelectrode distance. If volume conduction contributes to the coherence, a strong dependence on distance is to be expected. In contrast, specific neuronal interactions may or may not depend on distance. Although the measured coherence was on average higher for the smallest inves- tigated interelectrode distance $(\sim 600 \mu \mathrm{m})$ than for larger distances, we found no statistically significant effect of the electrode distance on the measured coherence averaged over the entire frequency range (Kruskal-Wallis multiple comparison ANOVA; $p=0.01$ ). This also holds true in analysis of single-frequency components of the LFP coherence. During the stimulus period, there is also no significant effect of the electrode distance for either the averaged coherence or single-frequency components. Therefore, it is very unlikely that volume conduction accounts for the measured LFP coherence. This result is in good accordance with the estimation of passive spread of the LFP reported earlier (Engel et al., 1990). Within the range of distances investigated, we found neither a significant effect of the interelectrode distance nor of the stimulus orientation on the peak frequency of the LFP coherence response (Kruskal-Wallis multiple comparison ANOVA; $p=0.01)$. However, there is a weak but highly significant correlation between the peak frequency of the LFP coherence response and the mean peak frequencies of LFP power response of the two recording sites for which the coherence is computed $\left(R^{2}=0.15 ; p<0.01 ; n=261\right)$.

Analogous to the analysis of orientation tuning of the LFP power, we computed the tuning index for the coherence between LFPs recorded simultaneously at different cortical sites. The tuning index for single-frequency bins and frequency bands was calculated for each pair of recording sites. For all pairs, the derived optimal frequency band was tested for significant orientation tuning $(p<0.01 ; n=211$; total $=512 ; 41 \%)$. The median of the derived frequencies of optimal orientation tuning was $48 \mathrm{~Hz}$ for $f_{\text {start }}(25$ th and 75th percentiles, 30 and $71 \mathrm{~Hz} ; n=211$ ) and 125 $\mathrm{Hz}$ for $f_{\text {stop }}$ (25th and 75th percentiles, 70 and $165 \mathrm{~Hz} ; n=211$ ). 
A

LFP coherence response
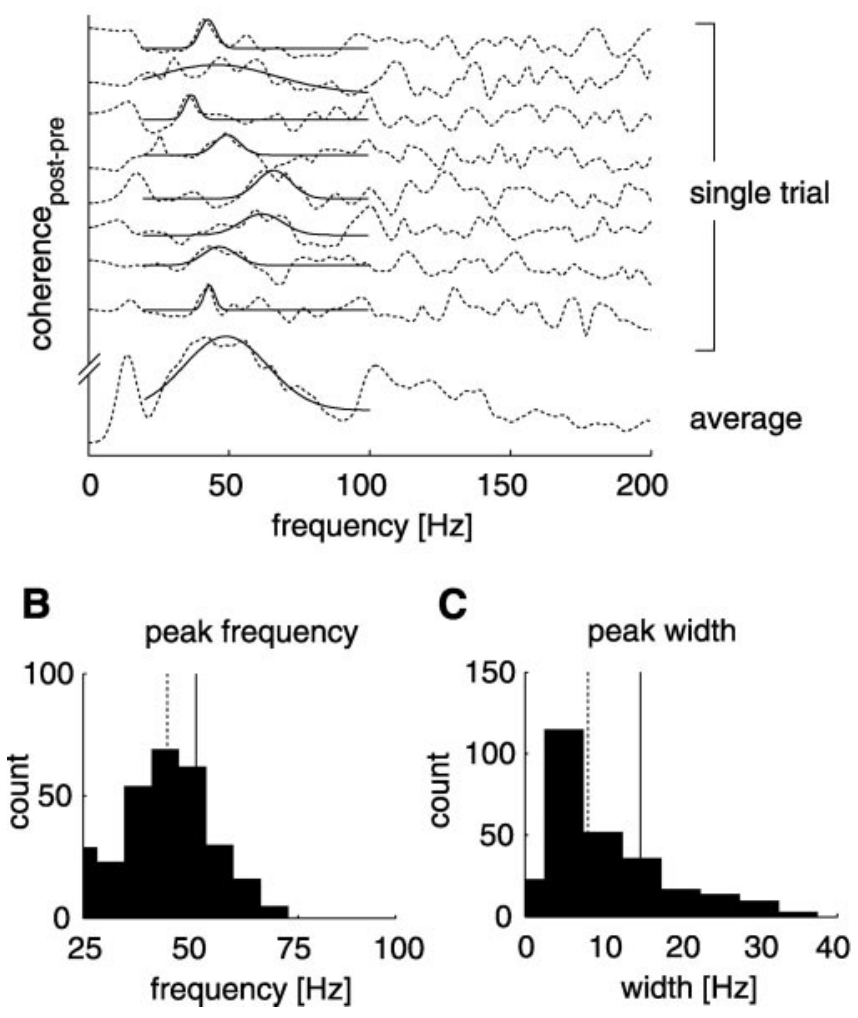

Figure 5. Single trial analysis of the LFP coherence response. $A$, The lower dashed trace shows the mean LFP coherence response averaged over all trials (same pair of recording sites and data set as in Fig. 4). The solid curve indicates the best fit of a Gaussian function over the frequency range of $25-100 \mathrm{~Hz}$. The upper eight dashed traces display the LFP coherence response of eight randomly chosen trials (same data set). The solid curves indicate the corresponding best Gaussian fits from $25-100 \mathrm{~Hz}$. B, Distribution of the peak frequencies of the Gaussian fits of all trials. The dashed line indicates the median of the distribution $(n=288$; median $=42 \mathrm{~Hz}$, same pair of recording sites). The solid line indicates the peak frequency of the Gaussian fit to the averaged LFP coherence response displayed in $A$ ( $49 \mathrm{~Hz}$ ). C, Distribution of the peak width of the Gaussian fits of all trials (SD of the fitted Gaussian). The dashed line indicates the median of the distribution ( $n=288$; median $=8.0 \mathrm{~Hz}$, same pair of recording sites). The solid line indicates the peak width of the Gaussian fit to the averaged LFP coherence response displayed in $A(14.8 \mathrm{~Hz})$.

Taking only single-frequency bins into account, the median of the optimal frequencies was $73 \mathrm{~Hz}$ (25th and 75th percentiles, 45 and $108 \mathrm{~Hz} ; n=211)$.

The averaged results of the frequency-dependent orientation tuning of the coherence for all significant pairs are shown in Figure 6. LFP coherence was tuned for stimulus orientation in a broad frequency range up to the highest investigated frequency of $200 \mathrm{~Hz}$, similar to short-range synchronization (Fig. 6A). However, in contrast to the tuning of short-range synchronization and the response of long-range synchronization, no distinct peak in low frequencies was observed. Only a small percentage of recording sites showed a distinct low-frequency band with significant orientation tuning $(p<0.01 ; n=20$; total $=211 ; 9 \%$; median $f_{\text {start }}=22 \mathrm{~Hz}$; median $f_{\text {stop }}=37 \mathrm{~Hz}$ ). Correspondingly, we find no local maximum in the averaged functions in the low-frequency range in Figure $6 B$.

In summary, visual stimulation induces an increase of longrange synchronization estimated by the LFP coherence in a broad frequency range. As observed for short-range synchronization, the frequency band for optimal orientation tuning is surprisingly high and does not include the $40 \mathrm{~Hz}$ bin. However, in contrast to

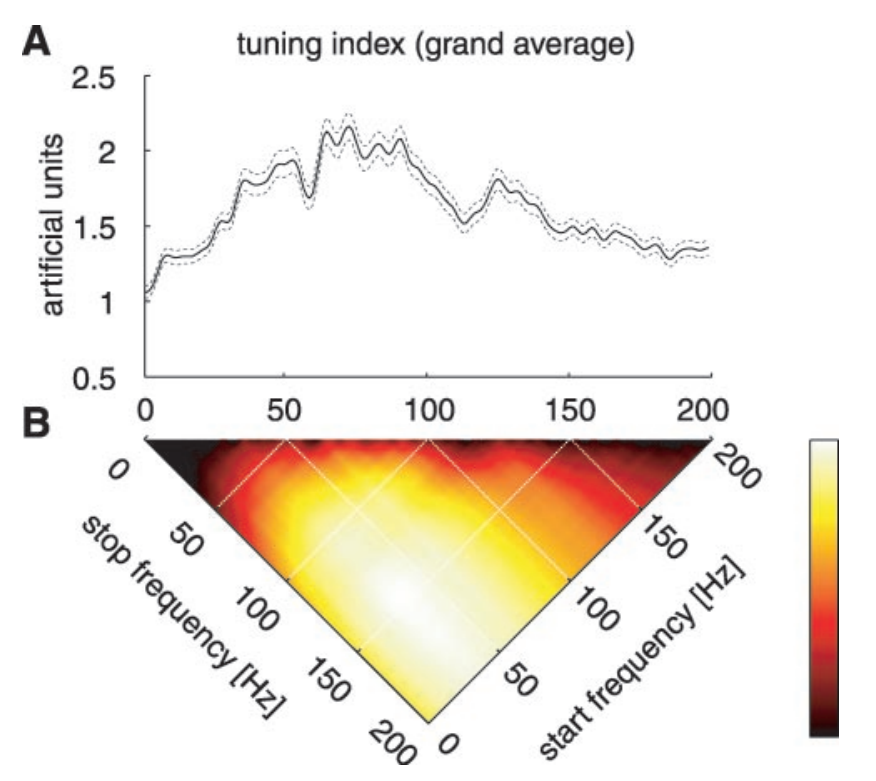

Figure 6. Grand average of the tuning parameters analyzed for the LFP coherence of all pairs of recording sites that showed a significant orientation tuning. $A$, Tuning index of the LFP coherence as a function of frequency $(n=211$; mean \pm SEM). $B$, Tuning index as a function of all possible frequency bands defined by $f_{\text {start }}$ and $f_{\text {stop }}(n=211)$. The function displayed in $A$ is identical to the values on the diagonal of $B$.

short-range synchronization, for long-range synchronization, visual stimulation also induces a distinct increase of synchronization in a low-frequency range $<30 \mathrm{~Hz}$.

\section{Frequency-dependent spike locking}

Because the study of LFPs allows no direct analysis of single-cell activity, we asked how these findings relate to the neuronal spiking behavior: are these frequency bands also reflected as a frequency-dependent synchronization of neurons to a pool of other neurons? To address these questions, we recorded MUA simultaneously with the LFP using the same stimulation protocol as described above ( $n=27$; total $=66 ; 41 \%$ of recording sites).

Figure $7 A$ shows the MUA peristimulus time histogram of a typical example with the transient on-response and the tonic part resulting from the relative motion of retina and the visual stimulus. Figure $7 B$ shows the single-trial raw LFP and MUA simultaneously recorded from the same recording site. Note the prominent increase of $\gamma$-oscillations and the increased spiking activity induced by visual stimulation. Figure $7 C$ shows an expanded view of the marked 200 msec epoch. The superimposed MUA trace reveals the strong tendency of neurons to spike during the peak negativity of the LFP $\gamma$-oscillation. To quantify the phase locking between the LFP and single spikes, we computed the STA for all pairs of MUAs and LFPs recorded at different sites separately for the delay and stimulus period. We averaged the STA of all pairs with a significant SFC (see below and Materials and Methods) response (Fig. $7 D)(p<0.01 ; n=244$; total $=415 ; 59 \%)$. During the delay period, the STA is rather flat. This indicates little phase locking of spikes to the LFP during spontaneous activity (Fig. 7D, solid trace). In contrast, during the stimulation period, a prominent oscillatory synchronization is visible between spiking activity and the LFP (Fig. 7D, dashed trace). The SFC (Fig. 7E), which corrects for changes in LFP power, shows two distinct peaks in the low- and high-frequency ranges during the stimulation period, whereas low frequencies dominate at a much lower level during the delay period. Accordingly, these two distinct high- and 
A

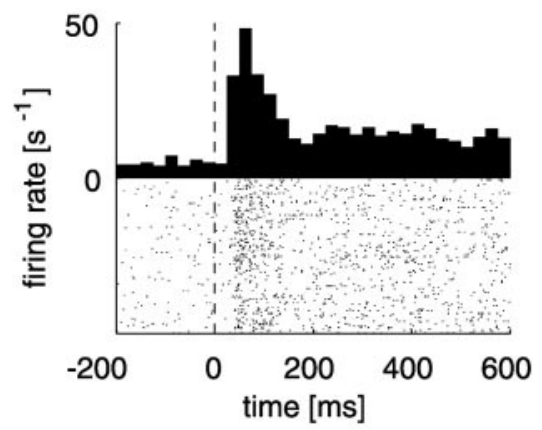

E

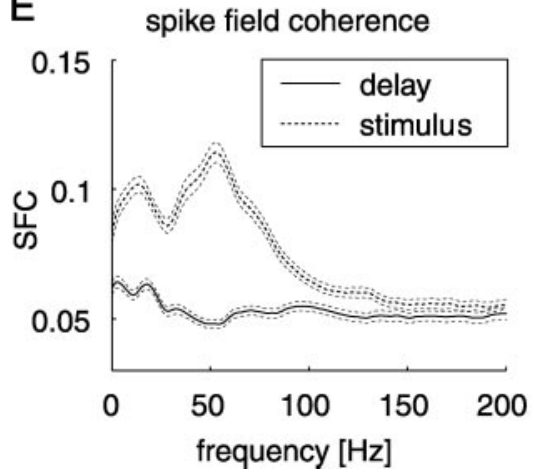

B

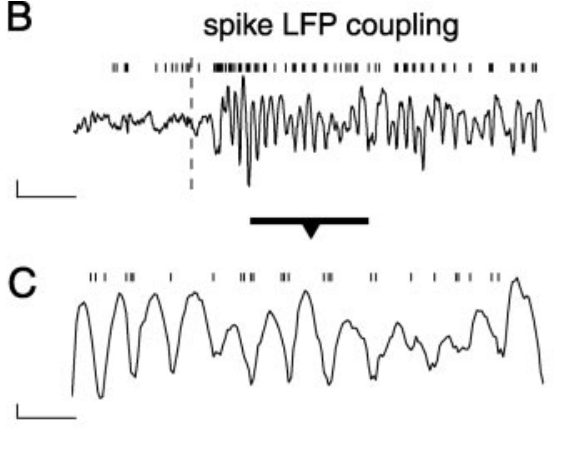

F

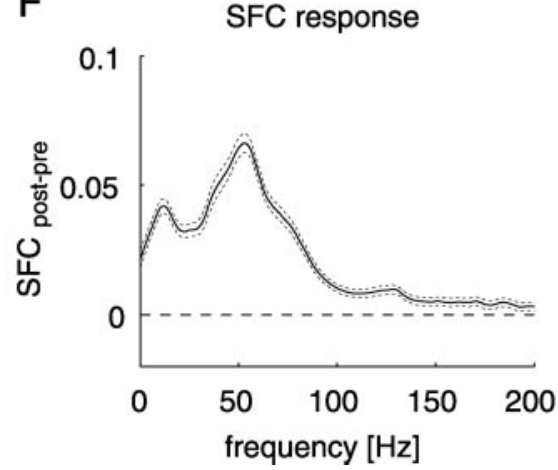

D

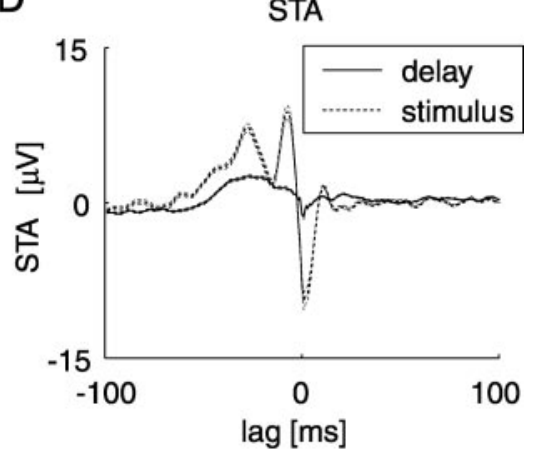

G

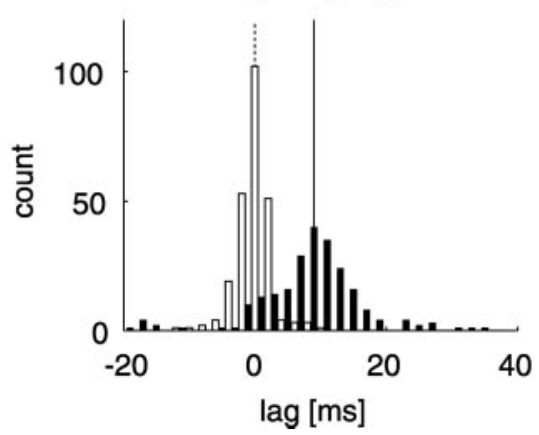

Figure 7. Frequency distribution of spike-LFP coupling. $A$, The peristimulus time histogram (PSTH) of multiunit activity (MUA) recorded at a typical recording site. The dashed line indicates stimulus onset. Ten single traces of all 12 stimulus orientations are shown beneath the histogram ( 25 msec binning). $B$, Single-trial raw LFP trace. The MUA recorded simultaneously at the same recording site is shown on top of the LFP trace. The 200 msec epoch marked by the horizontal bar is expanded below. The vertical dashed line indicates stimulus onset (horizontal scale bar, 100 msec; vertical scale bar, $100 \mu \mathrm{V}$ ). C, Expanded plot of the 200 msec epoch marked in B. A prominent phase locking between the LFP and the simultaneously recorded MUA is observed (horizontal scale bar, $25 \mathrm{msec}$; vertical scale bar, $50 \mu \mathrm{V}$ ). D, Grand average of spike triggered averages (STA) during delay and stimulus period over all recorded pairs of MUA and LFP that showed a significant spike field coherence (SFC) response ( $n=244$; mean \pm SEM). $E$, Grand average of the SFC during delay and stimulus period for the same pairs of recording sites ( $n=244$; mean \pm SEM). $F$, Grand average of the SFC response for all pairs of recording sites that showed a significant SFC response ( $n=244$; mean \pm SEM). $G$, The time lag of the maximum negativity was computed for the low-frequency $(1-23 \mathrm{~Hz})$ and high-frequency $(24-200 \mathrm{~Hz})$ components of all STAs of pairs of recording sites that showed a significant SFC response ( $n=244)$. The distribution of the lag for the high-frequency component is plotted as white bars (median $=0 \mathrm{msec}$; dashed line). The corresponding distribution for the low-frequency component is plotted as black bars (median $=10 \mathrm{msec}$; solid line).

low-frequency peaks are observed again in the average SFC response (Fig. $7 F$ ). Because the SFC is independent of spike rate and LFP power, the two peaks of the SFC during visual stimulation indicate a synchronization of neuronal spiking activity and neuronal activity at a different cortical site in two distinct frequency bands. Furthermore, observation of these two peaks in the SFC response as well shows that by visual stimulation, synchronization is specifically enhanced in these two frequency bands. The frequency distribution of the SFC during stimulation and the SFC response itself are in good accordance with the frequency distributions found by analyzing long- and short-range synchronization on the basis of the LFP power and LFP coherence. The median of low-frequency maxima of the SFC response is found at $12 \mathrm{~Hz}$, the median of high-frequency maxima at $53 \mathrm{~Hz}$. The dip between these two bands is found at $23 \mathrm{~Hz}$.

For comparison of the properties of these two frequency bands of the LFP, we asked whether there was a difference in the phase relationship between the LFP and spiking activity for the low- and high-frequency bands, respectively. Because in the STA (Fig. 7D) the high-frequency oscillation reaches its maximum negativity at $0 \mathrm{msec}$ lag, spikes are exactly phase-locked to this maximum negativity. In contrast, the negativity of the lowfrequency component is poorly aligned to $0 \mathrm{msec}$ lag. To quantify this difference, we filtered all STAs from 1 to $23 \mathrm{~Hz}$ and from 24 $\mathrm{Hz}$ to $200 \mathrm{~Hz}$ and computed separately the lag of the maximum negativity for the low- and high-frequency components (Fig. $7 G)$. The distribution of lags for the high-frequency band is cen- tered tightly around a median of $0 \mathrm{msec}$, whereas the distribution for the low-frequency band is broader, with a median of $10 \mathrm{msec}$. It should be noted that even when the lag is expressed in a suitably defined phase angle, the properties of the two frequency bands are markedly different. The distributions of lags show not only a difference in the width of distribution but also a significant difference in their median ( $p<0.01$; Wilcoxon test). In addition, it is plausible to assume that the functionally relevant time scale is more likely the absolute time (e.g., in milliseconds) than a normalized parameter like the phase angle.

\section{Discussion}

In the present study, we demonstrate stimulus-specific synchronization of neuronal activity in primary visual cortex of awake behaving cats. Visual stimulation induces a prominent broadband increase of short-range synchronization, long-range synchronization, and spike field coupling estimated by LFP power, LFP coherence, and SFC, respectively. In each case, the maximum increase of synchronization was observed in the range of 44-53 $\mathrm{Hz}$. The single frequency with optimally orientation-selective synchronization, however, was found at higher frequencies of $\sim 70 \mathrm{~Hz}$. Searching for a continuous band of optimal orientation tuning reveals a broad frequency range starting at $\sim 45 \mathrm{~Hz}$ and extending well beyond $100 \mathrm{~Hz}$. Remarkable are the qualitatively different properties of the distinct peak in the low-frequency range from $\sim 10$ to $20 \mathrm{~Hz}$. The analysis of the SFC confirms the results of the LFP analysis on the level of spiking activity. A broad 
distribution of high-frequency synchronization and synchronization in a low-frequency band is observed. Furthermore, the different phase lag of spike-field coupling in these two frequency bands suggests a distinct functional role of these two frequency bands.

A description of neuronal activity in different frequency bands has been used intensively in the study of different brain states. In some behavioral states, such as slow-wave sleep, low frequencies dominate. During reticular activation, the frequency distribution shifts to the $\gamma$-range (Munk et al., 1996; Steriade et al., 1996; Herculano-Houzel et al., 1999). Such stimulation occurs naturally during REM sleep, but similar phenomena might be observed during the depolarized phases of slow-wave sleep and the awake state (Steriade et al., 1996). Thus, the notion of "desynchronized brain states" is actually better described by a shift of rhythms to higher frequencies. It also has been shown that the frequency of fast oscillatory rhythms is correlated positively with the level of membrane depolarization (Steriade et al., 1996). Therefore, the broad frequency distribution of $\gamma$-oscillations and high intertrial variability found here might reflect the dynamics of varying membrane depolarization.

Given the interest in the $\gamma$-frequency band, the question of what the best choice of frequency band is has been surprisingly neglected. Here, we investigate the frequency distribution of neuronal synchronization. We do not question the definition of the $\gamma$-band as such but ask which high-frequency band in the $\gamma$-range can be derived considering objective criteria in an awake behaving animal. We build on the consideration that orientationselective neuronal activity is a characteristic property of primary visual cortex. This is applied to recordings in the primary visual cortex of awake cats under reasonably natural conditions. In another study of alert cats (Gray and Viana di Prisco, 1997), the animals were trained in a fixation task to suppress smooth pursuit of the stimulus in the region of the receptive field, a paradigm very similar to the ones used in many experimental studies of the monkey visual system (Tovee and Rolls, 1992; Frien et al., 1994; Kreiter and Singer, 1996; de Oliveira et al., 1997; König and Engel, 1995). In the present study, we also used a paradigm involving oriented stimuli moving relative to the retina, yet made an effort to adapt it to the behavioral repertoire of cats. We chose a tracking task, in which the effective visual stimulus served as a static background. Considering the time necessary to train a cat in a tracking compared with a fixation task, the former appears to be a relevant natural behavior. Using this more natural setting, we found stimulus-induced synchronization of neuronal activity in a broad frequency range extending well above $100 \mathrm{~Hz}$. The analysis of individual trials revealed a prominent trial-to-trial variability of the synchronization frequency that contributes to the broad frequency range over which synchronization is induced by visual stimulation. This is in good agreement with previous studies of alert cats (Gray and Viana di Prisco, 1997) and a large number of studies using anesthetized preparations (Singer and Gray, 1995). However, in the present study, the analysis of optimal orientation tuning resulted in a $\gamma$-band starting at $\sim 45 \mathrm{~Hz}$ and extending well above $100 \mathrm{~Hz}$. The decline of power with increasing frequency is not monotonous, however, but rather a secondary peak can be observed well beyond $100 \mathrm{~Hz}$. The bicoherence analysis shows a weak absolute phase coupling between these high-frequency components and slower $\gamma$-band rhythms that renders a harmonic cause of these local maxima very unlikely. Interestingly, such very-high-frequency activity ("ripples") was described recently to occur in various natural states of vigilance (Grenier et al., 2001). Furthermore, these authors re- port widespread synchronization of such rhythms, in line with the present results. Compared with the wide variety of choices for upper and lower boundaries of the frequency band investigated in previous studies, the resulting $\gamma$-band in the present investigation is surprisingly broad and located consistently at comparatively high frequencies.

These observations have several important implications. First, from a technical point of view, to improve the signal-to-noise ratio in physiological investigations, very-high-frequency activity, even $>100 \mathrm{~Hz}$, has to be considered. Furthermore, the activity integrated over the frequency band is a much better indicator than the peak frequency. Also, the lower cutoff should be placed high enough. In particular, centering the frequency band on the so-called " $40 \mathrm{~Hz}$ " seems not to be the optimal choice. Overall, these measures make experiments more readily comparable and allow observation of new aspects of cortical dynamics.

Second, the broad frequency distribution has implications for the mechanisms generating oscillatory activity in the $\gamma$-range. In previous theoretical studies, oscillatory units of different kinds have been used to model neuronal interactions (Sturm and König, 2001). Variations of the harmonic oscillator resulting from negative feedback of local inhibitory neurons or hyperpolarizing potassium currents tend to produce narrow frequency spectra, which do not match the results of the broad frequency distribution and the high trial-to-trial variability of the peak frequency found in the present study. Introducing strong sources of dynamic noise in the neural activity cannot reproduce the high trial-to-trial variability. Strong noise in the neuronal coupling, either dynamic or static, broadens the frequency distribution. However, they also make synchronization more unreliable. The results of the present investigation demonstrate that orientationselective synchronization occurs even at very high frequencies. Considering transmission delays of cortical interactions, this poses tight constraints on the mechanisms involved. It is not clear that the types of models proposed are compatible with this finding.

A third important aspect relates to the interactions of different frequency bands. The spiking activity of individual neurons couples to the population activity with zero phase lag in the $\gamma$-band. In contrast, the low-frequency components show a significant phase lag to the spiking activity. This is compatible with two different functional roles of activity in the low- and highfrequency bands, respectively: synchronization in the $\gamma$-range has been implicated with binding of distributed neuronal activity to coherent representation of visual stimuli. Although a considerable number of experiments in different species and cortical systems have been performed, this hypothesis is still intensely debated, and the final verdict is not yet in (Singer, 1999; Shadlen and Movshon, 1999). Nevertheless, it has been shown that functional coupling in the striate cortex of the awake monkey shows stronger stimulus dependency for oscillations in the $\gamma$-range than for low-frequency components (Frien and Eckhorn, 2000; Frien et al., 2000). Furthermore, differential synchronization in the lowfrequency range and the $\gamma$-range in different cortical areas of awake cats and monkeys has recently been related to the context of a behavioral task and the expectancy of the experimental subject (von Stein et al., 2000; Fries et al., 2001; Engel et al., 2001). Using a theoretical approach, these experimental data can be understood in the context of bottom-up and top-down interactions, taking into account the asymmetric morphology of cortical pyramidal neurons (Siegel et al., 2000). In pyramidal neurons, top-down signals targeting the apical dendritic tree are integrated separately from afferent signals targeting the basal dendritic tree 
(Larkum et al., 1999; Körding and König, 2000). Depending on the behavioral context of the current sensory processing, this differential integration of different pathways can lead to characteristic synchronization patterns in the low- and high-frequency ranges. This is in good accordance with the specific spike field coupling of neurons observed in the two different frequency bands. Thus, the two frequency bands defined here may relate to distinct cortical mechanisms integrating bottom-up and topdown information.

\section{References}

Abeles M (1982) Local circuits: an electrophysiological study. Berlin: Springer.

Brosch M, Bauer R, Eckhorn R (1997) Stimulus-dependent modulations of correlated high-frequency oscillations in cat visual cortex. Cereb Cortex 7:70-76.

Chrobak JJ, Buzsaki G (1998) Gamma oscillations in the entorhinal cortex of the freely behaving rat. J Neurosci 18:388-398.

de Oliveira SC, Thiele A, Hoffmann KP (1997) Synchronization of neuronal activity during stimulus expectation in a direction discrimination task. J Neurosci 17:9248-9260.

Destexhe A, Contreras D, Steriade M (1999) Spatiotemporal analysis of local field potentials and unit discharges in cat cerebral cortex during natural wake and sleep states. J Neurosci 19:4595-4608.

Eckhorn R (1994) Oscillatory and non-oscillatory synchronizations in the visual cortex and their possible roles in associations of visual features. Prog Brain Res 102:405-426.

Engel AK, König P, Gray CM, Singer W (1990) Stimulus-dependent neuronal oscillations in cat visual cortex: inter-columnar interaction as determined by cross-correlation analysis. Eur J Neurosci 2:588-606.

Engel AK, Fries P, Singer W (2001) Dynamic predictions: oscillations and synchrony in top-down processing. Nat Rev Neurosci 2:704-716.

Fell J, Klaver P, Lehnertz K, Grunwald T, Schaller C, Elger CE, Fernandez G (2001) Human memory formation is accompanied by rhinal-hippocampal coupling and decoupling. Nat Neurosci 4:1259-1264.

Frien A, Eckhorn R (2000) Functional coupling shows stronger stimulus dependency for fast oscillations than for low-frequency components in striate cortex of awake monkey. Eur J Neurosci 12:1466-1478.

Frien A, Eckhorn R, Bauer R, Woelbern T, Kehr H (1994) Stimulus-specific fast oscillations at zero phase between visual areas V1 and V2 of awake monkey. NeuroReport 5:2273-2277.

Frien A, Eckhorn R, Bauer R, Woelbern T, Gabriel A (2000) Fast oscillations display sharper orientation tuning than slower components of the same recordings in striate cortex of the awake monkey. Eur J Neurosci 12:1453-1465.

Fries P, Roelfsema PR, Engel AK, König P, Singer W (1997) Synchronization of oscillatory responses in visual cortex correlates with perception in interocular rivalry. Proc Natl Acad Sci USA 94:12699-12704.

Fries P, Reynolds JH, Rorie AE, Desimone R (2001) Modulation of oscillatory neuronal synchronization by selective visual attention. Science 291:1560-1563.

Gray CM, Viana di Prisco G (1997) Stimulus-dependent neuronal oscillations and local synchronization in striate cortex of the alert cat. J Neurosci 17:3239-3253.

Grenier F, Timofeev I, Steriade M (2001) Focal synchronization of ripples $(80-200 \mathrm{~Hz})$ in neocortex and their neuronal correlates. J Neurophysiol 86:1884-1898.

Hatsopoulos NG, Harrison MT, Donoghue JP (2001) Representations based on neuronal interactions in motor cortex. Prog Brain Res 130:233-244.
Herculano-Houzel S, Munk MH, Neuenschwander S, Singer W (1999) Precisely synchronized oscillatory firing patterns require electroencephalographic activation. J Neurosci 19:3992-4010.

König P, Engel AK (1995) Correlated firing in sensory-motor systems. Curr Opin Neurobiol 5:511-519.

Körding KP, König P (2000) Learning with two sites of synaptic integration. Network 11:25-39.

Kreiter AK, Singer W (1996) Stimulus-dependent synchronization of neuronal responses in the visual cortex of the awake macaque monkey. J Neurosci 16:2381-2396.

Kruse W, Eckhorn R (1996) Inhibition of sustained gamma oscillations $(35-80 \mathrm{~Hz})$ by fast transient responses in cat visual cortex. Proc Natl Acad Sci USA 93:6112-6117.

Larkum ME, Zhu JJ, Sakmann B (1999) A new cellular mechanism for coupling inputs arriving at different cortical layers. Nature 398:338-341.

Llinas R, Ribary U (1993) Coherent 40-Hz oscillation characterizes dream state in humans. Proc Natl Acad Sci USA 90:2078-2081.

Martinez-Conde S, Macknik SL, Hubel DH (2000) Microsaccadic eye movements and firing of single cells in the striate cortex of macaque monkeys. Nat Neurosci 3:251-258.

Miltner WH, Braun C, Arnold M, Witte H, Taub E (1999) Coherence of gamma-band EEG activity as a basis for associative learning. Nature 397:434-436.

Munk MH, Roelfsema PR, König P, Engel AK, Singer W (1996) Role of reticular activation in the modulation of intracortical synchronization. Science 272:271-274.

Ohl FW, Scheich H, Freeman WJ (2001) Change in pattern of ongoing cortical activity with auditory category learning. Nature 412:733-736.

Press WH, Teukolsky SA, Vetterling WT, Flannery BP (1992) Numerical recipes: the art of scientific computing, Ed 2. Cambridge, MA: Cambridge UP.

Rodriguez E, George N, Lachaux JP, Martinerie J, Renault B, Varela FJ (1999) Perception's shadow: long-distance synchronization of human brain activity. Nature 397:430-433.

Shadlen MN, Movshon JA (1999) Synchrony unbound: a critical evaluation of the temporal binding hypothesis. Neuron 24:67-77.

Siegel M, Körding KP, König P (2000) Integrating top-down and bottom-up sensory processing by somato-dendritic interactions. J Comput Neurosci 8:161-173.

Singer W (1999) Neuronal synchrony: a versatile code for the definition of relations? Neuron 24:49-65.

Singer W, Gray CM (1995) Visual feature integration and the temporal correlation hypothesis. Annu Rev Neurosci 18:555-586.

Steriade M (1999) Coherent oscillations and short-term plasticity in corticothalamic networks. Trends Neurosci 22:337-345.

Steriade M, Amzica F, Contreras D (1996) Synchronization of fast (30-40 $\mathrm{Hz}$ ) spontaneous cortical rhythms during brain activation. J Neurosci 16:392-417.

Sturm AK, König P (2001) Mechanisms to synchronize neuronal activity. Biol Cybern 84:153-172.

Swindale NV (1998) Orientation tuning curves: empirical description and estimation of parameters. Biol Cybern 78:45-56.

Tallon-Baudry C, Bertrand O, Delpuech C, Permier J (1997) Oscillatory gamma-band (30-70 Hz) activity induced by a visual search task in humans. J Neurosci 17:722-734.

Tovee MJ, Rolls ET (1992) Oscillatory activity is not evident in the primate temporal visual cortex with static stimuli. NeuroReport 3:369-372.

Varela F, Lachaux JP, Rodriguez E, Martinerie J (2001) The brainweb: phase synchronization and large-scale integration. Nat Rev Neurosci 2:229-239.

von Stein A, Chiang C, König P (2000) Top-down processing mediated by interareal synchronization. Proc Natl Acad Sci USA 97:14748-14753. 